\title{
Wound healing complications after revascularization for moyamoya vasculopathy with reference to different skin incisions
}

\author{
Güliz Acker, MD, ${ }^{1,2}$ Nicolas Schlinkmann, ${ }^{1}$ Lucius Fekonja, MAD, ${ }^{1,3}$ Lukas Grünwald, ${ }^{1}$ \\ Juliane Hardt, Dipl-Psych, ${ }^{4,5}$ Marcus Czabanka, MD, ${ }^{1}$ and Peter Vajkoczy, MD ${ }^{1}$
}

1Department of Neurosurgery and Center for Stroke Research Berlin, Charité-Universitätsmedizin Berlin (corporate member of Freie Universität Berlin, Humboldt-Universität zu Berlin, and Berlin Institute of Health), Berlin; ${ }^{2}$ Berlin Institute of Health, Berlin; ${ }^{3}$ Cluster of Excellence: "Image Knowledge Gestaltung: An Interdisciplinary Laboratory," Humboldt University, Berlin; ${ }^{4}$ Institute of Biometry and Clinical Epidemiology, Charité-Universitätsmedizin Berlin (corporate member of Freie Universität Berlin, Humboldt-Universität zu Berlin, and Berlin Institute of Health), Berlin; and ${ }^{5} \mathrm{Clinical}$ Research Unit, Berlin Institute of Health, Berlin, Germany

OBJECTIVE Moyamoya vasculopathy (MMV) is a steno-occlusive cerebrovascular disease that can be treated by a surgical revascularization. All the revascularization techniques influence the blood supply of the scalp, with a risk for wound healing disorders. The authors' aim was to analyze the wound healing process in the patients who underwent a direct or combined bypass surgery with a focus on different skin incisions.

METHODS The authors retrospectively identified all the patients with MMV who were treated surgically in their institution. Subsequently, they analyzed demographic data, clinical symptoms, surgical treatment, and detailed history of complications. Based on the evolution of their surgical techniques and the revascularization strategy to be used, the authors applied the following skin incisions: linear incision, curved incision, incomplete $Y$ incision, and complete $Y$ incision Group comparisons regarding wound healing disorders were performed with significance testing using Fisher's exact test.

RESULTS The authors identified 172 patients with MMV (61.6\% moyamoya disease, $7 \%$ unilateral moyamoya disease, $29.7 \%$ moyamoya syndrome, and 1.7\% unilateral moyamoya syndrome), of whom 124 underwent bilateral operations. One-quarter of the patients were juveniles. A total of 236 hemispheres were included in the analysis, of which $27.9 \%$ were treated by a combined procedure with encephalomyosynangiosis. Overall, $5.1 \%$ major and $1.7 \%$ minor wound complications occurred. The overall wound complication rate was lower in direct revascularization compared to combined revascularization ( $3 \%$ vs $15.2 \%$ ). The lowest incidence of wound healing disorders was found in the linear incision group for the parietal superficial temporal artery branch (1.6\%), followed by the incomplete $Y$ incision group for the frontal branch of the superficial temporal artery (3.8\%) in the direct bypass group. In the combined revascularization cohort, major or minor wound disorders appeared in $14.3 \%$ and $4.8 \%$, respectively, in the complete $Y$ incision group and in $4.2 \%$ (for both major and minor) in the curved incision group. The complete $Y$ incision caused significantly more wound healing disorders compared to the remaining incision types (17.1\% vs $3.1 \%, p=0.007$ ).

CONCLUSIONS Wound healing disorders are one of the major complications of revascularization surgery. Their incidence depends on the revascularization strategy and skin incision applied, with a complete $Y$ incision giving the worst results.

https://thejns.org/doi/abs/10.3171/2018.11.FOCUS18512

KEYWORDS moyamoya disease; STA-MCA; superficial temporal artery-middle cerebral artery; EMS;

encephalomyosynangiosis; revascularization

ABBREVIATIONS EDAMS = encephaloduroarteriomyosynangiosis; EDAS = encephaloduroarteriosynangiosis; EMS = encephalomyosynangiosis; $M C A=$ middle cerebral artery; MMD = moyamoya disease; MMS = moyamoya syndrome; MMV = moyamoya vasculopathy; STA = superficial temporal artery.

SUBMITTED September 29, 2018. ACCEPTED November 15, 2018.

INCLUDE WHEN CITING DOI: 10.3171/2018.11.FOCUS18512. 
$\mathrm{M}$ OYAMOYA vasculopathy (MMV) is a rare cerebrovascular disease characterized by bilateral progressive steno-occlusion of basal cerebral arteries with coexisting abnormal netlike vessels ${ }^{15}$ The most frequent initial symptom in adults with moyamoya disease (MMD) varies depending on the patient's ethnicity and their age at disease onset. ${ }^{3,4,14}$ Intracranial hemorrhage is the leading initial symptom in Asian adult patients, whereas ischemic events predominate in Caucasian adult patients and in juvenile MMV patients worldwide. ., $^{3,14}$

A randomized clinical trial demonstrated revascularization surgery as the most effective treatment for hemorrhagic MMV. ${ }^{13}$ Although randomized trials have not so far been published for ischemic MMV, based on various studies, revascularization surgery could be the most effective treatment in the management of patients with MMD regardless of type of the disease, to reduce ischemic and hemorrhagic stroke rate and improve clinical outcome. ${ }^{2,9}$ Various surgical techniques have been described, including 3 different revascularization strategies: indirect, direct, and combined revascularization. Our recent review focused on the effectiveness of the different revascularization strategies for management of MMV and concluded that a direct bypass should be applied to patients with MMD, if technically feasible. ${ }^{2}$ A combined revascularization strategy is recommended especially for juvenile patients, in whom the direct bypass failure rate and the indirect bypass take rate are higher. ${ }^{2,6}$

Wound healing disorders including skin necrosis, infection, or CSF leakage are common complications of direct or combined revascularization surgery due to impaired blood supply to the scalp, reaching up to $31 \% .{ }^{5,16,19}$ The impairment of the scalp microcirculation is not only due to the harvesting of one of the superficial temporal artery (STA) branches, but also due to the impairment of the scalp microvascular network by the preparation of the skin flap. Conventionally, a large skin-galea flap with a separate muscle is prepared using a curved skin incision for a pterional craniotomy for the combined revascularization surgery (STA-middle cerebral artery [MCA] combined with encephalomyosynangiosis [EMS]), as described for an STA-MCA bypass and encephaloduroarteriosynangiosis (EDAS) combined procedure. ${ }^{11}$ The crucial role of the galeal incision and the vessel harvesting techniques in wound-related complications has already been discussed in the literature. ${ }^{16}$ Less invasive techniques for the flap preparation, such as a Y-like incision (interrupted or incomplete $\mathrm{Y}$ incision), have previously been described for direct bypass surgery with STA-MCA anastomosis, including double-barrel bypass procedures. ${ }^{10,18}$

Over the past decade, we went through different stages of surgical evolution in our department, applying different surgical techniques and concomitant skin incisions for direct and combined revascularization procedures, including linear, curved, interrupted $\mathrm{Y}$, and complete $\mathrm{Y}$ skin incisions. Hence, the aim of our study was to analyze wound healing complications in our patients with respect to different skin incision techniques after direct and combined revascularization surgeries.

\section{Methods \\ Study Design}

Retrospective analysis of patient data was approved by the local human healthcare ethics committee. We retrospectively identified all MMV patients, including those with unilateral moyamoya and quasi-moyamoya, who presented to our department between January 2007 and June 2018 using ICD code I67.5 (moyamoya). MMD, quasi-MMD, and unilateral MMD were defined based on the guidelines established by the Research Committee for Moyamoya Disease; accordingly, patients with a possible associated disease were classified as quasi-MMD. ${ }^{14}$ Patients with atherosclerosis were excluded from the analysis. We gathered data about patients' characteristics regarding disease type and treatment, clinical outcome, operative technique, and complications. We included all the surgically treated patients with a minimum of one follow-up examination at least 3 months after surgery in the analysis. We analyzed the wound healing disorders, including dehiscence, infection, necrosis, and CSF leakage, with respect to different skin incision techniques. We defined wound disorders requiring a surgical revision as major wound disorders and the less severe cases that were treated only conservatively (antibiotics, lumbar drainage, etc.) as minor wound disorders. Subgaleal hemorrhage was reported separately.

\section{Different Skin Incisions}

Following our preoperative evaluation of the donor vessels, we favor one of the STA branches for the direct bypass part and plan the incision based on this. Depending on the preferred STA branch in case of a sole direct revascularization, we perform a linear incision for the parietal branch and an incomplete $\mathrm{Y}$ incision for the frontal branch of the STA. For a combined revascularization, we routinely perform a curved incision for the parietal branch or a complete $\mathrm{Y}$ incision for the frontal branch of the STA.

For combined revascularization (STA-MCA combined with EMS), we performed a curved incision that is extended frontally to enable a pterional craniotomy if the parietal STA branch was used as donor vessel (Figs. 1A and 2A), and a complete Y-shaped incision (Figs. 1B and 2B) if the frontal branch of the STA was used as donor vessel. For the latter, a first skin incision was made along the frontal STA branch, followed by a second skin incision above the ear, ending in the previous incision and forming a "complete" Y (Figs. 1B and 2B). This technique reduces the skin flap area and enables a faster mobilization of the frontal STA branch. The subsequent surgical steps were the same for all procedures. A separate skin-muscle flap was prepared. The muscle was cut in a curved fashion and mobilized. After a subperiosteal preparation, a pterional craniotomy was performed. Subsequently, the direct bypass was established, and the temporal muscle was positioned on the brain surface and sutured to the dura mater, resulting in an EMS. For direct revascularization with an STA-MCA anastomosis, we applied a linear skin incision if the parietal STA branch was used as donor vessel (Figs. 

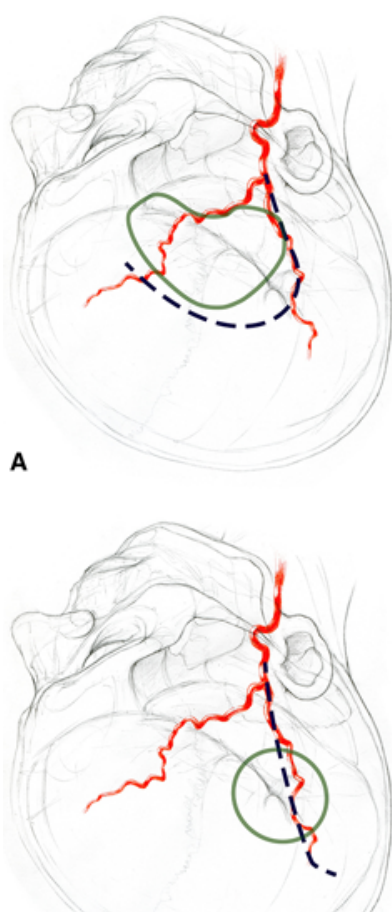

C

FIG. 1. Illustration of the reported skin incisions for combined and direct revascularization procedures with the craniotomy (green). The flap method with a curved incision (A) and a complete Y-shaped incision (B) with a pterional craniotomy for the bypass with the STA-MCA anastomosis and EMS. A linear incision (C) and an incomplete $Y$ incision (D) with targeted craniotomy for the STA-MCA bypass.

$1 \mathrm{C}$ and $2 \mathrm{C}$ ), and an incomplete $\mathrm{Y}$ skin incision if the frontal branch of STA was used as donor vessel. For the latter, a first skin incision was made along the frontal STA branch, followed by a separate second skin incision above the ear in order to allow a targeted craniotomy at the end of the sylvian fissure, resulting in an incomplete $\mathrm{Y}$ incision to provide better wound healing than a complete Y-shaped incision (Figs. 1D and 2D). Here, the bypass vessel is tunneled below the remaining skin bridge and transposed to the anastomosis site. This technique enables-as mentioned above - a faster and atraumatic mobilization of the frontal STA branch without a large skin flap and without mobilization of supraorbital skin/galea.

\section{Statistical Analysis}

This study shows descriptive analyses and group comparisons: numbers and percentages of surgical revascularization techniques applied and of wound healing complications. For the group of patients with wound healing disorders, it additionally shows the configurations of the sociodemographic and clinical characteristics of the patients and several aspects of the treatments (operative vs conservative or lumbar drain). All analyses were done with Excel (version 14.7.7; Microsoft) and SPSS (version 25; IBM Corp.). Group comparisons analyzed the proportion of patients with wound healing complications 1) for operations with bypass versus combined bypass with
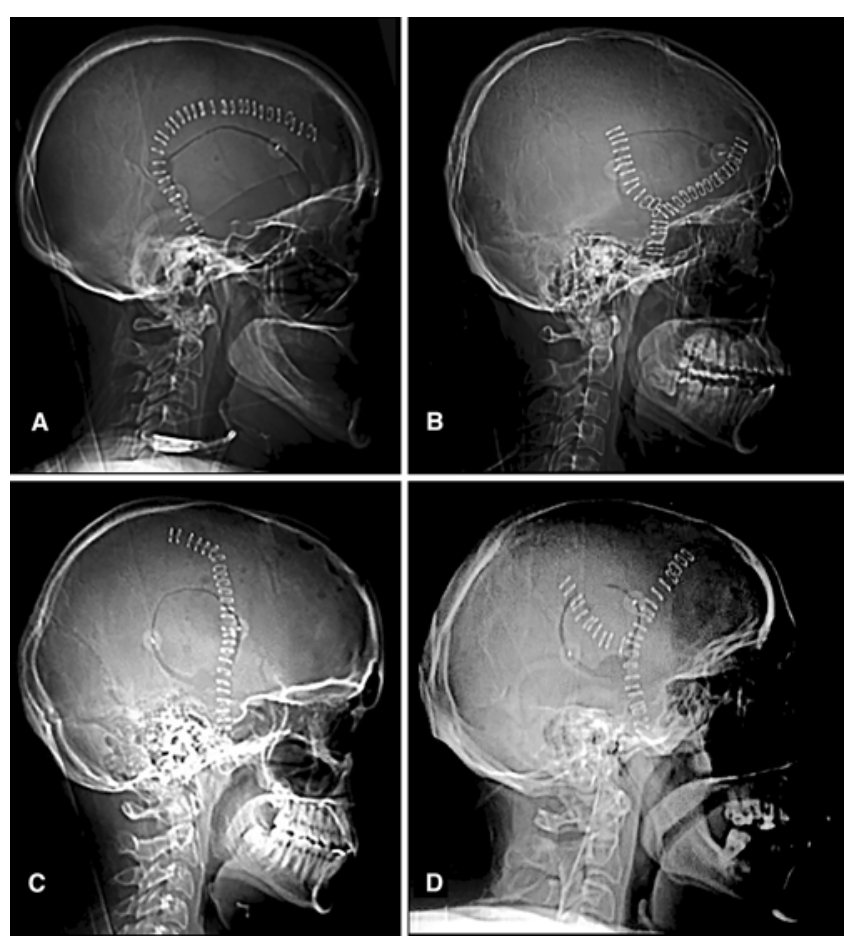

FIG. 2. Skull radiographs demonstrating the craniotomy in relation to the incisions illustrated in Fig. 1. The flap method with a curved incision (A) and a complete Y-shaped incision (B) with a pterional craniotomy for the bypass with the STA-MCA anastomosis and EMS. A linear incision (C) and an incomplete $Y$ incision (D) with targeted craniotomy for the STAMCA bypass.

EMS; 2) for Y-shaped versus other incision forms; 3) for Y-shaped versus curved incisions; and 4) for MMD versus moyamoya syndrome (MMS). Significance testing was done with Fisher's exact test. Because data for the first and second hemispheres are dependent, the statistical analyses were done for all patients with an operation on the first hemispheres, without including the second hemispheres in the analyses.

\section{Results}

A total of 235 patients with MMV were identified, of whom 213 were surgically treated at our institution. We excluded 41 patients due to the lack of follow-up visits. Of the remaining $172 \mathrm{MMV}$ patients included in the analysis, $61.6 \%$ had MMD, $7 \%$ had unilateral MMD, $29.7 \%$ had MMS, and $1.7 \%$ had unilateral MMS. One-quarter of the patients were juvenile. Ischemic events represented the major onset symptom (79.7\%), hemorrhagic MMV was only found in $9.3 \%$ of the cases, and the remaining onset symptoms were headache, seizure, dizziness, and cognitive disorders. The majority of the patients were Caucasians (87\%).

A total of 124 of 172 patients underwent bilateral operations; 1 patient's first surgery was done in another hospital, and 47 hemispheres had to be excluded due to missing follow-ups for the second surgery, resulting in a total of 248 hemispheres for the analysis. Table 1 summarizes the different surgical techniques. Eleven hemispheres that were treated by only an indirect revascularization tech- 
TABLE 1. Summary of all surgical revascularization techniques applied in our patient cohort divided between first and second hemisphere

\begin{tabular}{lccc}
\hline $\begin{array}{c}\text { Revascularization } \\
\text { Technique }\end{array}$ & $\begin{array}{c}\text { 1st } \\
\text { Hemisphere, } \\
\mathrm{n}=171\end{array}$ & $\begin{array}{c}\text { 2nd } \\
\text { Hemisphere, } \\
\mathrm{n}=77\end{array}$ & $\begin{array}{c}\text { Total, } \\
\mathrm{n}=248\end{array}$ \\
\hline Indirect & 4 & 7 & 11 \\
\hline Direct & 110 & 60 & 170 \\
\hline Combined w/ EMS & 55 & 10 & 65 \\
\hline Double bypass w/ EMS & 1 & 0 & 1 \\
\hline Intermediate-flow bypass & 1 & 0 & 1 \\
\hline Total & 171 & 77 & 248 \\
\hline
\end{tabular}

nique and the 1 intermediate bypass were also excluded from further analysis. A total of 236 hemispheres with direct or combined revascularization with STA-MCA bypass and EMS were included in the final analysis (Fig. 3). The majority of the hemispheres were treated by an STAMCA bypass only ( $72 \%$ direct bypass vs $27.9 \%$ combined with EMS). In the direct bypass cohort, an incomplete Y incision or a linear incision was performed in $63 \%$ and $36.5 \%$ of the cases, respectively, depending on the harvested STA branch. In the combined revascularization cohort, the frontal branch of the STA was harvested for bypass surgery by using a continuous Y-shaped incision in $63.6 \%$. The remaining $36.4 \%$ of the hemispheres were treated using the flap technique with a curved incision, in which the frontal branch of the STA was used only in 3 cases in which the parietal branch was intraoperatively not suitable, and in 1 case both STA branches were taken for a double-barrel bypass procedure.

In all 236 hemispheres, a total of $5.1 \%$ and $1.7 \%$ showed major and minor wound healing problems, respectively. A subgaleal hemorrhage occurred only in $0.8 \%$ of all cases (Table 2). Of the patients with a wound disorder, $43.8 \%$ were juvenile (Table 3). In 1 patient the first hemisphere treated by STA-MCA combined with EMS in which a complete $\mathrm{Y}$ incision was used suffered from a superficial wound dehiscence. However, an infection and CSF leakage occurred in the second hemisphere, which was treated with STA-MCA with an incomplete $\mathrm{Y}$ incision. This patient had MMS with thrombophilia and trisomy 21. None of the patients with a wound complication suffered from diabetes mellitus. Overall, the patients who received treatment by only a direct bypass surgery via targeted craniotomy showed a lower wound complication rate, including major and minor wound disorders, compared to the rate for the combined revascularization procedure with EMS (via pterional craniotomy) in our patient cohort (3\% vs $15.2 \%$ ). The lowest wound complication rate was $1.6 \%$ with a linear incision for the direct bypass procedure, followed by the incomplete $\mathrm{Y}$ incision with a wound complication rate of $3.8 \%$ (Table 2). In the hemispheres that were treated using a curved incision, wound complications were observed in $8.4 \%$. In contrast, the highest wound complication rate, amounting to $19 \%$, was observed when using a complete Y-shaped incision. In 1 case a subgaleal hemorrhage occurred, which also had to be revised operatively.

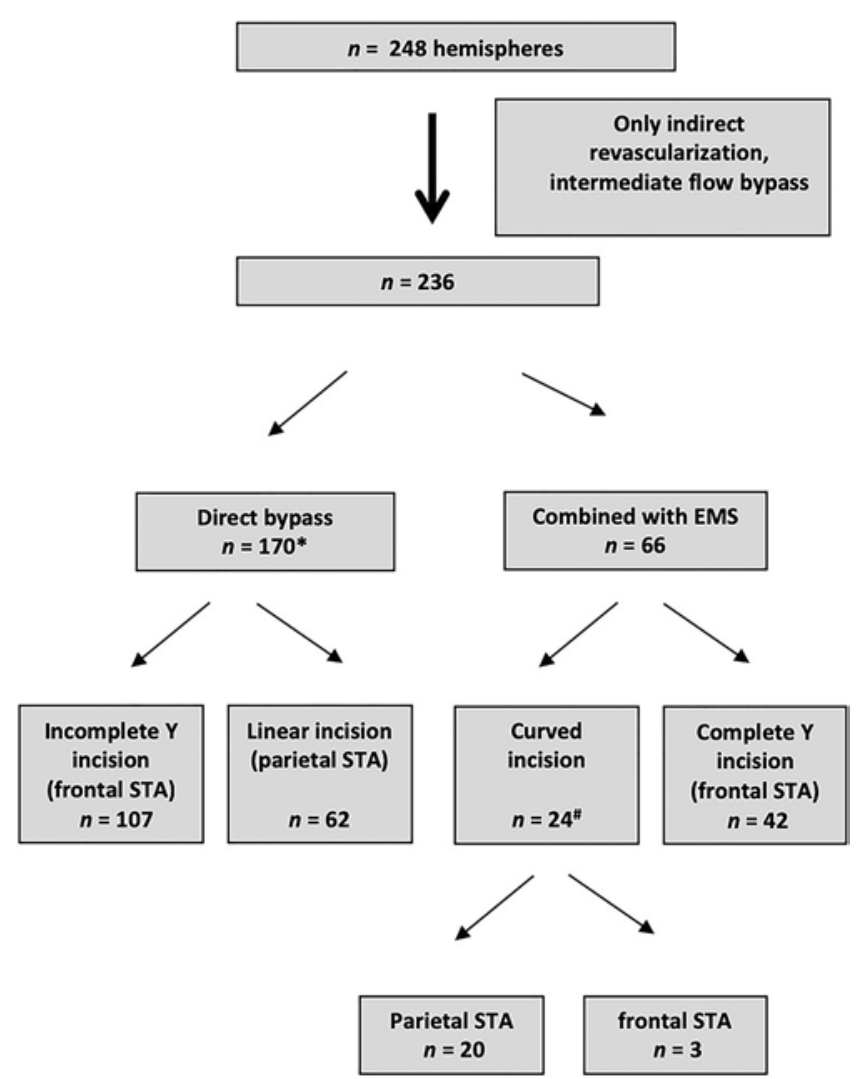

FIG. 3. A flowchart of the direct or combined surgically treated hemispheres including different incision techniques. *One patient had a previous hemicraniotomy and received a bypass through the previous incision. \#One patient was surgically treated via double direct bypass, harvesting both STA branches.

Our statistical analyses of all the patients with regard to the first surgically treated hemispheres (first side: $\mathrm{n}=171$ ) confirmed the lower proportions of wound healing complications in the direct revascularization group reported above versus a combined revascularization with EMS ( $\mathrm{n}=$ 2 [1.8\%] vs $n=8$ [14.3\%], $p=0.003$ ). Furthermore, we detected significantly higher proportions of wound healing disorders for complete Y-shaped incision versus all other incision types in the first hemisphere $(n=6[17.1 \%]$ vs $n=$ $4[3.1 \%], p=0.007)$. The direct comparison of Y-shaped incision versus curved incision in combined procedures showed a tendency toward a higher event rate of wound healing disorder $(n=6[17.1 \%]$ vs $n=2[10 \%])$; however, due to the low sample size the difference was not significant $(\mathrm{p}=0.696)$. The analysis of the diagnosis (MMD vs MMS) did not show any significant differences for the occurrence of wound healing problems $(p=1.00)$.

The occurrence time of the wound disorders varied between 6 and 154 days after surgery (Table 3). An occlusion of STA-MCA bypass occurred in 3 patients. Removal of the bone was required in 5 cases. In 6 cases, a bacterial infection was diagnosed in the surgically obtained microbiological sample. In one patient 2 surgical revisions were needed, and in another patient 3 surgical revisions were needed. 
TABLE 2. Summary of all wound disorders in 236 hemispheres that underwent either a direct bypass surgery or a combined revascularization with STA bypass and EMS listed according to the skin incisions

\begin{tabular}{|c|c|c|c|c|c|c|c|}
\hline Procedure & No. & No. w/ Major WHD & $\%$ & No. w/ Minor WHD & $\%$ & No. w/ Galeal Hemorrhage & $\%$ \\
\hline Linear & 62 & 1 & 1.6 & & & & \\
\hline Direct bypass after previous hemicraniotomy & 1 & 1 & & & & & \\
\hline \multicolumn{8}{|l|}{ Combined w/ EMS via pterional craniotomy } \\
\hline Total & 236 & 12 & 5.1 & 4 & 1.7 & 2 & 0.8 \\
\hline
\end{tabular}

WHD = wound healing disorder.

\section{Discussion}

The overall wound complication rate at our institution (5.1\% major and $1.7 \%$ minor wound complications) was at the lower edge of the range reported in the literature. ${ }^{16,19}$ The principal novel finding of our study is that combined revascularization using a complete Y-shaped incision tends to cause more wound complications.

Surgical revascularization strategies for the patients with MMV led to impairment of the blood supply of the scalp, causing wound healing disorders at a widely ranging rate $(0.7 \%$ up to $31.7 \%))^{1,5,16,19}$ The majority of these studies did not report the skin incision technique in particular. For instance, in 1997 Houkin et al. reported a $1.78 \%$ wound healing disorder rate in 112 hemispheres that were surgically treated by STA-MCA bypass combined with encephaloduroarteriomyosynangiosis (EDAMS) without specification of the skin incision. ${ }^{7}$ Mesiwala et al. detected wound disorders in $5 \%$ of 65 revascularization procedures including 56 direct bypasses, also without further information about the branch of the STA or skin incision used..$^{12} \mathrm{~A}$ recent study by Zhao et al. in which they reported on 610 hemispheres including $12.7 \%$ direct and $10 \%$ combined revascularization procedures without further information about the skin incision showed an overall impaired wound healing rate of $2 \%$ of their cases. ${ }^{20}$ In the 2011 European patient cohort with a combined revascularization STAMCA and EMS, a wound healing disorder was reported in $8 \%$ of the cases. ${ }^{6}$

For better comparability with our data, we focused on studies with information about the skin incisions. For instance, Abla et al. reported $0.7 \%$ wound healing disorders in 140 treated hemispheres that underwent a direct bypass surgery with the parietal branch of the STA using a linear incision, which was comparable to our wound complication rate using the same type of incision. ${ }^{1}$ Takanari et al. analyzed 98 hemispheres $(86.7 \%$ were surgically treated by a direct or combined revascularization with EDAS or EDAMS) with a wound complication rate of $21.4 \%$, also including minor wound disorders without a need for surgical intervention. ${ }^{16}$ This study indicated diabetes mellitus as well as double-bypass type procedures defined as 2 STA bypasses, combined surgery (STA-MCA with EDAMS or EDAS), or a combination of 2 indirect procedures as risk factors. The authors interpreted different skin inci- sion techniques with regard to blood supply, suggesting a "linear curved line" incision as a safe procedure, similar to our linear incision technique. Incomplete or complete $\mathrm{Y}$ incisions were not reported in the Takanari et al. study. Although a linear incision seems to be the safest, Chung et al. recently reported a rate of $39.1 \%$ for skin necrosis after direct bypass, using a linear incision along the course of the artery, where in $29 \%$ of the cases the frontal branch of the STA was harvested. ${ }^{5}$ The authors observed less skin necrosis, with a rate of $22.2 \%$ using a curvilinear incision approximately $2 \mathrm{~cm}$ behind the donor artery and an in-toout dissection technique of the donor artery. However, the difference was not significant between both groups. Although the rates for skin necrosis reported for both groups were high, the authors highlighted the fact that no wound infections occurred, probably due to the preservation of the fibrous septa and galeal aponeurotica.

There are several different revascularization surgery techniques, and because the definition for wound healing disorders differs among the above-mentioned studies, it is difficult to compare them. Our recent review including several meta-analysis studies did not find any studies showing a correlation between performed revascularization techniques (direct, indirect, and combined) and wound healing disorders. ${ }^{2}$ Consequently, it is a wellknown fact that wound healing disorder is a serious and relevant complication of revascularization surgery, regardless of the revascularization technique itself. Several new surgical techniques have been proposed to reduce wound healing disorders, including endoscopic procedures for the dissection of both STA branches or different dissection techniques for the STA. ${ }^{5,17}$ Chung et al. indicated that an in-to-out dissection of the STA could protect against wound infection. ${ }^{5}$ A less invasive incomplete Y-shaped incision has also previously been described for direct bypass surgery for STA-MCA anastomosis..$^{10,18}$

Katsuta et al. compared different skin incision techniques called "flap method" and "cut-down" method in an arteriosclerotic patient population. They concluded that the less invasive cut-down method with Y incision might be preferable to avoid wound disorders; however, there was not a significant difference between the groups. ${ }^{10}$ Yoshimura et al. also described an incomplete Y-shaped incision for a double bypass without any wound complications in a small patient cohort. ${ }^{18}$ In our population of 


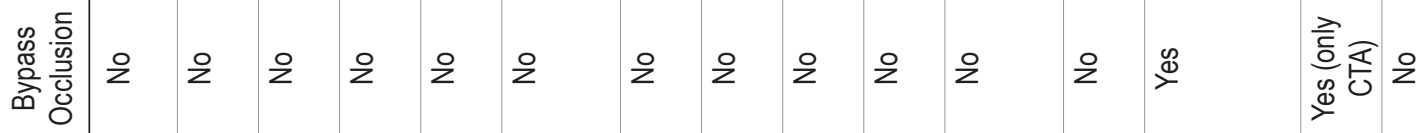

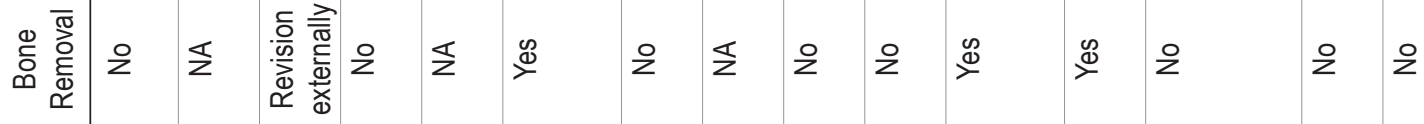

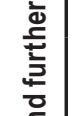

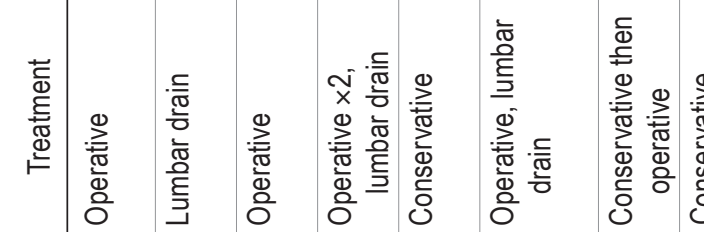

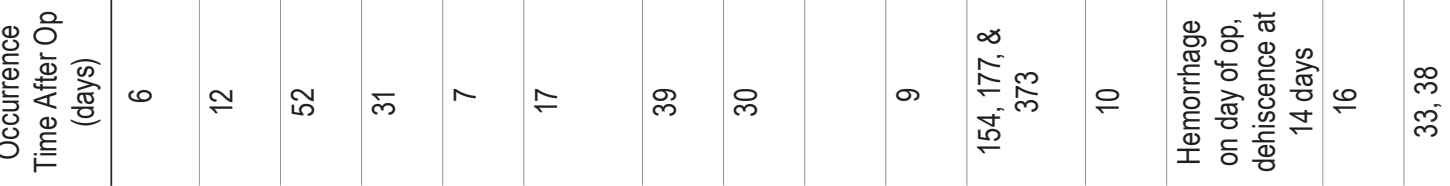

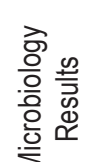

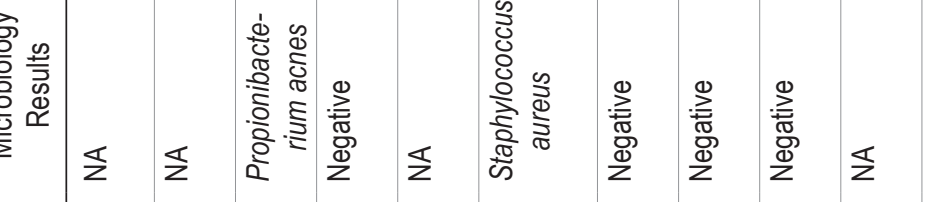

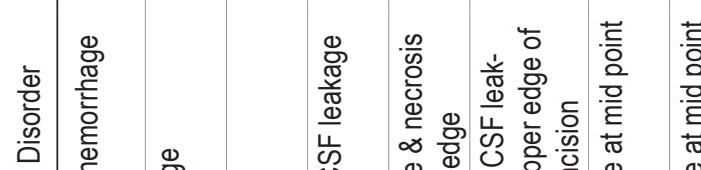

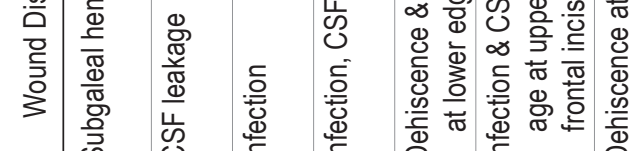

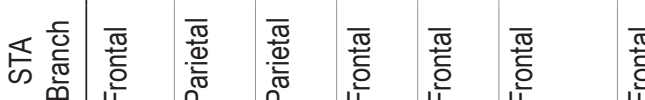

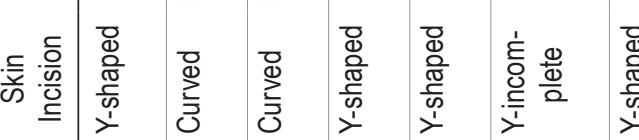

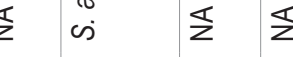

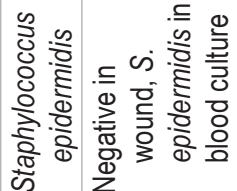

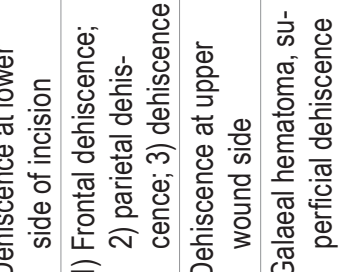

$\stackrel{\circ}{\circ}$

恶 ब용 
patients with incomplete $\mathrm{Y}$ skin incision, the major wound complication rate was also low, at $2.8 \%$. A complete Yshaped incision is a well-established technique for temporoparietal fascial flap elevation for versatile reconstructive procedures. ${ }^{3,4}$ Interestingly, there were no wound healing disorders in an analysis of 57 temporoparietal fascial flaps (aside from alopecia and hematoma), including those performed using the Y-shaped incision technique. ${ }^{3}$

Although there are several incision techniques besides the Y-shaped incision (such as lazy S, inverted T, or zigzag techniques), one advocated advantage of the Y-shaped incision for flap preparation is the variable dimension due to the adjustable size of the vertical incision and wide or narrow bifurcation angle. ${ }^{3}$ To our knowledge, a continuous Y-shaped incision technique has so far not been reported for STA-MCA bypass with EMS requiring a pterional craniotomy. We established this technique to enable a faster and less invasive dissection of the frontal STA branch, which otherwise has to be located in the conventional flap technique. With the help of a second skin incision above the ear, the mobilization of the temporal muscle and a pterional craniotomy could be performed easily. Although the rate for major wound complications (14.3\%) was within the reported range, this appears to be higher than for other skin incisions among our patients. The preparation and harvesting of the temporal muscle with subperiosteal preparation might play an important role for the wound healing process. In this regard, the most suitable comparison can be made with the curved incision technique for the pterional craniotomy, for which the major wound complication rate was $4.2 \%$ among our patients. However, because the number of patients (n $=24 ; 20$ cases with the parietal branch, 1 with both STA branches) was low in this group, the question of whether the harvested branch of STA (frontal vs parietal) plays a key role in wound complications cannot be answered sufficiently. Imai et al. reported a rate of $2.3 \%$ for severe infectious wound complication resulting in bone removal after using an L-shaped incision similar to the curved incision for a combined revascularization with the parietal branch of STA for adults. ${ }^{8}$ This is comparable to our complication rate in adults in whom a curved incision is used. Importantly, Imai et al. did not report less severe wound complications in detail. ${ }^{8}$ Because the children were partly treated only with an indirect revascularization strategy, a comparison to our juvenile population could not be made.

Almost half of our affected patients were juvenile (43.8\%), and none of the patients with a wound disorder had diabetes mellitus. Half of the affected patients had MMS, with comorbidities including trisomy 21, thrombophilia, vasculitis, microcephaly, and Graves' disease. However, due to the low number of patients an association cannot be made to determine whether MMS patients would be at higher risk for wound infections. In our population, for direct bypass the linear and incomplete $\mathrm{Y}$ incisions both led to a low percentage of wound disorders. Among all revascularization procedures, the complete $\mathrm{Y}$ incision caused significantly more wound healing disorders. The comparison of the complete with incomplete $\mathrm{Y}$ incision (14.3\% vs $2.8 \%$ major wound healing disorder) suggested that the meeting point of the $\mathrm{Y}$ in the complete version may represent a weak point, possibly leading to 
more wound disorders. However, the localization of the wound disorders was not limited to this edge point in our cohort. Based on our findings, a complete Y incision technique cannot be recommended unconditionally and should only be applied in selected cases. It is also important to emphasize that we only included patients in whom a combination with EMS was performed for combined revascularization. Therefore, higher wound complications after combined revascularization cannot be generalized for all combined procedures, including less invasive techniques such as encephalodurosynangiosis (EDS).

\section{Study Limitations}

The limitation of this study is that it was a retrospective analysis within a single institution. However, this is the first study describing a complete $\mathrm{Y}$ incision technique for a combined revascularization strategy.

\section{Conclusions}

Our study represents the first report focusing on wound complications with comparison of different skin incisions including complete and incomplete $\mathrm{Y}$ incision for revascularization techniques in MMV. The complete $\mathrm{Y}$ incision technique seems to be associated with more wound healing disorders-thus further applications should be limited.

\section{Acknowledgments}

Dr. Acker is a participant in the BIH-Charite Clinician Scientist Program funded by the Charité-Universitätsmedizin Berlin and the Berlin Institute of Health.

\section{References}

1. Abla AA, Gandhoke G, Clark JC, Oppenlander ME, Velat GJ, Zabramski JM, et al: Surgical outcomes for moyamoya angiopathy at Barrow Neurological Institute with comparison of adult indirect encephaloduroarteriosynangiosis bypass, adult direct superficial temporal artery-to-middle cerebral artery bypass, and pediatric bypass: 154 revascularization surgeries in 140 affected hemispheres. Neurosurgery 73:430-439, 2013

2. Acker G, Fekonja L, Vajkoczy P: Surgical management of moyamoya disease. Stroke 49:476-482, 2018

3. Acker G, Goerdes S, Schneider UC, Schmiedek P, Czabanka M, Vajkoczy P: Distinct clinical and radiographic characteristics of moyamoya disease amongst European Caucasians. Eur J Neurol 22:1012-1017, 2015

4. Baba T, Houkin K, Kuroda S: Novel epidemiological features of moyamoya disease. J Neurol Neurosurg Psychiatry 79:900-904, 2008

5. Chung Y, Lee SH, Choi SK: Fundamental basis of scalp layering techniques to protect against wound infection: a comparative study between conventional and in-to-out dissection of the superficial temporal artery. World Neurosurg 97:304-311, 2017

6. Czabanka M, Peña-Tapia P, Scharf J, Schubert GA, Münch E, Horn P, et al: Characterization of direct and indirect cerebral revascularization for the treatment of European patients with moyamoya disease. Cerebrovasc Dis 32:361-369, 2011

7. Houkin K, Ishikawa T, Yoshimoto T, Abe H: Direct and indirect revascularization for moyamoya disease surgical techniques and peri-operative complications. Clin Neurol Neurosurg 99 (Suppl 2):S142-S145, 1997

8. Imai H, Miyawaki S, Ono H, Nakatomi H, Yoshimoto Y, Saito N: The importance of encephalo-myo-synangiosis in surgical revascularization strategies for moyamoya disease in children and adults. World Neurosurg 83:691-699, 2015
9. Jeon JP, Kim JE, Cho WS, Bang JS, Son YJ, Oh CW: Metaanalysis of the surgical outcomes of symptomatic moyamoya disease in adults. J Neurosurg 128:793-799, 2018

10. Katsuta T, Inoue T, Arakawa S, Uda K: Cutaneous necrosis after superficial temporal artery-to-middle cerebral artery anastomosis: is it predictable or avoidable? Neurosurgery 49:879-884, 2001

11. Kuroda S, Houkin K: Bypass surgery for moyamoya disease: concept and essence of surgical techniques. Neurol Med Chir (Tokyo) 52:287-294, 2012

12. Mesiwala AH, Sviri G, Fatemi N, Britz GW, Newell DW: Long-term outcome of superficial temporal artery-middle cerebral artery bypass for patients with moyamoya disease in the US. Neurosurg Focus 24(2):E15, 2008

13. Miyamoto S, Yoshimoto T, Hashimoto N, Okada Y, Tsuji I, Tominaga T, et al: Effects of extracranial-intracranial bypass for patients with hemorrhagic moyamoya disease: results of the Japan Adult Moyamoya Trial. Stroke 45:1415-1421, 2014

14. Research Committee on the Pathology and Treatment of Spontaneous Occlusion of the Circle of Willis: Guidelines for diagnosis and treatment of moyamoya disease (spontaneous occlusion of the circle of Willis). Neurol Med Chir (Tokyo) 52:245-266, 2012

15. Suzuki J, Takaku A: Cerebrovascular "moyamoya" disease. Disease showing abnormal net-like vessels in base of brain. Arch Neurol 20:288-299, 1969

16. Takanari K, Araki Y, Okamoto S, Sato H, Yagi S, Toriyama $\mathrm{K}$, et al: Operative wound-related complications after cranial revascularization surgeries. J Neurosurg 123:1145-1150, 2015

17. Tamura Y, Aoki A, Yamada Y, Nonoguchi N, Yagi R, Tucker A, et al: Dissection of both frontal and parietal branches of the superficial temporal artery for bypass surgery through a single linear skin incision. Acta Neurochir (Wien) 153:1645-1648, 2011

18. Yoshimura S, Egashira Y, Enomoto Y, Yamada K, Yano H, Iwama T: Superficial temporal artery to middle cerebral artery double bypass via a small craniotomy: technical note. Neurol Med Chir (Tokyo) 50:956-959, 2010

19. Yu J, Shi L, Guo Y, Xu B, Xu K: Progress on complications of direct bypass for moyamoya disease. Int J Med Sci 13:578-587, 2016

20. Zhao M, Deng X, Zhang D, Wang S, Zhang Y, Wang R, et al: Risk factors for and outcomes of postoperative complications in adult patients with moyamoya disease. J Neurosurg [epub ahead of print March 30, 2018. DOI: 10.3171/2017.10. JNS171749]

\section{Disclosures}

The authors report no conflict of interest concerning the materials or methods used in this study or the findings specified in this paper.

\section{Author Contributions}

Conception and design: Vajkoczy, Acker. Acquisition of data: Acker, Schlinkmann, Grünwald. Analysis and interpretation of data: Vajkoczy, Acker, Hardt. Drafting the article: Vajkoczy, Acker, Fekonja. Critically revising the article: Vajkoczy, Schlinkmann, Fekonja. Reviewed submitted version of manuscript: Vajkoczy, Schlinkmann, Fekonja, Grünwald, Hardt, Czabanka. Approved the final version of the manuscript on behalf of all authors: Vajkoczy. Statistical analysis: Hardt. Administrative/technical/material support: Czabanka. Study supervision: Vajkoczy. Figures: Fekonja.

\section{Correspondence}

Peter Vajkoczy: Charité-Universitätsmedizin Berlin, Germany. peter.vajkoczy@charite.de. 